\title{
New Frontiers of Service Robotics for Active and Healthy Ageing
}

\author{
Alessandro Di Nuovo $^{1}$ - Frank Broz ${ }^{2}$ - Filippo Cavallo ${ }^{3}$ Paolo Dario ${ }^{3}$
}

Published online: 3 May 2016

(C) Springer Science+Business Media Dordrecht 2016

The world's population is growing old and the care of the elderly is becoming one of the great challenges for all the most developed regions (e.g. USA, Europe, Japan, Australia). It was estimated that, by the year 2050 , there will be three times more people over the age 85 than there are today and more than $20 \%$ of the population of western countries will be 65 and over. This demographic trend will lead to a growing number of older people living alone and in need of (intensive) care, but, at the same time, more financially well-appointed and wealthy senior citizens ready to enjoy their third age.

All these factors are leading an increased need for services for ageing well, which is pushing the growth of service robotics market to new frontiers, requiring new research progress and increasing industrial and social interest and commitment. The domain of service robotics for supporting independent living, addressed by home robots with a high degree of adaptability and autonomy in decision-making, has requirements which clearly go significantly beyond those of industrial robotics.

The ambition of this emerging domain is to provide robotic services with a high level of performance and acceptability. The complexity of the required services necessitates sophisticated and novel approaches to in order to fulfil them. These approaches may include multi-robot cooperation or the support of an Ambient Intelligence (AmI) infrastructure. The robotic systems developed face challenging real-world environments and may need to operate in both domestic and

Alessandro Di Nuovo

a.dinuovo@shu.ac.uk

1 Sheffield Robotics, Department of Computing, Sheffield Hallam University, 153 Arundel Street, Sheffield S1 2NU, UK

2 School of Mathematical and Computer Sciences, Heriot-Watt University, Edinburgh, UK

3 Biorobotics Institute, Scuola Superiore Sant'Anna, Pisa, Italy urban contexts. These systems also face unique challenges in usability and human-robot interaction. Interfaces must be designed to facilitate the operation of robots by the elderly, provide effective tools to supervise services and ensure safe operation, and connect users (elderly people and caregivers), service providers (social services, medical centres, municipalities, shops, pharmacies, etc.) and robots (indoor and outdoor).

In this perspective, the present special issue gives a multidisciplinary view of the most recent approaches and solutions for the general feasibility, the scientific and technological effectiveness, the social, ethical and legal plausibility, and the acceptability by end-users of a variety of complete advanced robotic services for elderly people in the home, communal shared living areas, and outdoor environments.

Many of the papers published here in this special issue are the result of the intensive international collaboration, in particular in Europe. Project funded by the European Commission, such as the Robot-Era project (http://www. robot-era.eu), are advancing the technology and the integration of robotic platforms within ambient assisted living environments, implementing a plurality of services using a user-centred approach and demonstrating how such an approach can build usable and acceptable services that can actively work in real conditions and cooperate with real people to provide favourable independent living, improving the quality of life and the efficiency of care for elderly people.

The first paper, titled Increasing the Acceptance of Assistive Robots for Older People Through Marketing Strategies Based on Stakeholder Needs, presents the results of an investigation on how to increase currently low acceptance of assistive robots through marketing strategies that address needs and fears of the different stakeholders (e.g. as users, decision makers and buyers). The authors present the results 
of a user-centered approach of identifying stakeholders and marketing strategies by analyzing different needs in an iterative design process from an early stage with quantitative and qualitative methods. The study identifies functionality, usability, safety, costs and financing, (non-) stigmatization and ethical aspects as the most important acceptance factors identified for assistive robotics.

In the second article of the issue, Enabling Global Robot Navigation Based on aCloud Robotics Approach, the authors describe a novel system, based on a cloud robotics paradigm, which is conceived to improve the navigation capability of autonomous robots in indoor environments that are not known a priori. The algorithm divides the environment into sub-maps and, then, all the necessary information and the topological representation of the world are stored in a remote cloud infrastructure. Next, by means of specific environmental tags, composed of a set of ARTags and QR codes, the robot can detect its location, access to the cloud service to retrieve the information, and update its navigation configuration in a dynamic and automatic way.

The third article, On robots and insurance, provides the reader with an overview of risk management of robotic applications through insurance contracts. The authors discuss the essential need of insurance products for an effective technology transfer from research to market in the field of service robotics. This article discusses the hindrances and identifies the essential questions that lawyers, economists, and engineers need to address in their future research in order to overcome current limitations and ultimately develop efficient and adequate risk management tools.

In the fourth paper, A Cloud Robotics solution to improve Social Assistive Robots for Active and Healthy Aging, authors suggest that recent advances in cloud technologies can open new opportunities for the provisioning of advanced robotic services. In particular, the manuscript investigates how the cooperation of a system of many connected robots, smart environments, and other devices can be improved by the huge computational and storage capability of cloud technologies. The cloud robotic services were tested in two realistic environments to assess the general feasibility of the proposal and demonstrate the ability to provide assistive location-based services in multiple environment frameworks.

The fifth article, titled Can a service robot which supports independent living of older people disobey a command? The views of older people, informal carers and professional caregivers on the acceptability of robots, focus on one ethical implication of autonomous robots. Through focus group meetings, Authors explored the areas of tension and the boundaries of a re-enablement robot coach for older people. What became apparent is that potential users are open to the idea of having a robot to support them in their daily life. Moreover, the concept of a robot performing higher level coordinating tasks within the role of a re-enablement coach demonstrated to be acceptable by the elderly participants of the study.

The sixth article, Long-term Evaluation of a Telepresence Robot for the Elderly-Methodology and ecological case study, proposes a methodology in the area of elderly people support, called MARTA (Multidimensional Assessment of telepresence RoboT for older Adults) for the long-term study of telepresence robot's adaptivity and compatibility in the field of support to social interaction of elderly people. The paper also presents a one year-long validation study during which a telepresence robotic platform (Giraff) has been deployed and iteratively assessed. Finally, the authors provide remarks on the technology readiness and suggestions for its improvements, while they encourage pursuing a multi-disciplinary user-centered approach for service design and assessment.

Generally speaking, the contributions in this special issue suggest that future research in service robotics for the elderly should be multidisciplinary, involving scientists from all the different disciplines (engineers, computer scientists, medicine doctors, sociologists, psychologists, lawyers, professional caregivers, economists, etc...), and it should include users, carers and all potential stakeholders and decision makers in the loop. Besides technological limitations of the current prototypes, there are mostly ethical and social, organizational and market-driven barriers identified.

The potential application fields of assistive robots for elderly are evident, but the services and business models must be compatible with existing structures and should be evaluated with that focus. Indeed, it is evident to us that future research in service robotics for the elderly should focus on the needs of potential stakeholder, especially of undeveloped countries, because there are different economic structures resulting in different needs.

Furthermore, considering a futuristic scenario, in which robots will be able to move autonomously in Smart Cities, it is clear that the Cloud represents a powerful mechanism that allows the sharing of the knowledge. With the proposed system, heterogeneous robots can dynamically access information about the environment through artificial landmarks.

We believe that the major priority in future should be spreading the knowledge that robots can be a useful instrument for ageing well to overcome some scepticism that has been reported, especially in the western countries. This could be achieved through more effective communication between science and industry and by incorporating mass media.

Acknowledgments We wish to express our gratitude to editor-in-chief Shuzhi Sam Ge, who supported us to prepare this special issue, as well as to all the reviewers for their valuable contribution in assuring that the selected papers met the high standard of the journal. 